\title{
Reversible multiple cranial nerve palsies in temporal (giant cell) arteritis with normal sedimentation rate: Report of a case
}

\author{
FREDERICK G. FREITAG, DO \\ SEYMOUR DIAMOND, MD \\ GLEN D. SOLOMON, MD, FACP \\ Chicago, Illinois
}

\begin{abstract}
Variations of giant cell arteritis present a difficult situation for the clinician. The Westergren sedimentation rate is usually elevated at the onset of the process and returns to normal values subsequent to treatment. In certain cases, the clinical manifestations of the disease provide the sole criterion for diagnosis and treatment. Because the neurologic sequela of giant cell arteritis are considered irreversible, prompt and agressive therapy with corticosteroids is essential for a successful outcome. The following is a case presentation of a patient demonstrating both of these dilemmas.
\end{abstract}

Temporal (giant cell) arteritis is easily recognized in its classic form. Since the first report of this disorder by Horton and associates ${ }^{1}$ in 1932 , it has become evident that it may have many different variations and symptoms of presentation. Classically, the disease is associated with significant elevations of the erythrocyte sedimentation rate (ESR). However, in $8 \%$ to $30 \%$ of patients who are diagnosed as having temporal arteritis, the ESR is found to be normal. ${ }^{2}$

Up to $50 \%$ of patients with temporal arteritis experience visual symptoms. ${ }^{3}$ These usually involve loss of vision, although other visual distur- bances, such as diplopia and ophthalmoplegia, may occur in $12 \%-15 \%$ of cases. ${ }^{4}$ These complications generally are considered to be irreversible. However, Lipton and coworkers ${ }^{5}$ recently reported a case of visual loss in temporal arteritis that resolved with the use of corticosteroids.

In the case reported here, the patient presented with a normal ESR. His visual loss, ophthalmoplegia, and diplopia all were reversed by corticosteroid therapy.

\section{Report of case}

A 75-year-old white man with a long history of chronic cluster headache visited the Diamond Headache Clinic on a emergency basis. He complained of a new type of headache, which had begun some 16 hours earlier. The patient stated that his pain, which he described as severe yet distinctly different from his chronic headaches, was located in the right eye and temporoparietal area. He was unable to wear a hat because of pain intensification. He had taken medication consisting of isometheptene, dichlorphenazone, and acetaminophen (Midrin) without relief, as well as acetominophen with $60 \mathrm{mg}$ of codeine on three occasions without benefit. He was also taking theophylline for treatment of chronic bronchitis, and imipramine and dipyridamole for the treatment of his cluster headaches.

Physical, neurologic, and ophthalmoscopic examinations were essentially normal. The fundoscopic examination revealed minimal arterial narrowing at the arterial venous crossings in both eyes. The right superficial 
temporal artery was firm and tender to palpation; the pulse was palpable in the underlying artery. The patient's ESR was determined in the office to be $5 \mathrm{~mm} / \mathrm{h}$, Westergren method (normal, 0-20 $\mathrm{mm} / \mathrm{h}$ ).

Arrangements were made for right superficial temporal artery biopsy 3 days later, and corticosteroid therapy (methylprednisone, $32 \mathrm{mg}$ per day) was begun. The patient was instructed to report any change in the headache or new symptoms, including changes in vision, at once. Over the next few days, there was a slight reduction in pain.

On the morning of the scheduled biopsy, the patient's wife called from the hospital while the patient was in surgery to report that he had developed double vision the night before. The attending surgeon was contacted and the patient was sent directly after his outpatient surgery to us for examination, which revealed the development of diplopia secondary to right cranial nerve III and VI palsies.

The patient was admitted to the hospital, and the steroid therapy was changed to prednisone ( $60 \mathrm{mg}$ per day) pending the results of the biopsy. The Westergren ESR at this point was $3 \mathrm{~mm} / \mathrm{h}$. The hemoglobin value was $17.1 \mathrm{~g} / \mathrm{dL}$. The complete blood count, SMA-12 battery, and immunoglobin electrophoresis showed normal results. Selective four-vessel angiography demonstrated only minimal atherosclerotic changes in the common carotid arteries, and computed tomographic scans of the head were normal. The biopsy report revealed marked narrowing of a portion of the right superficial temporal artery, and granulomatous changes with typical giant cells were seen within the intima of the artery.

There was slow recovery of third cranial nerve function over the subsequent week. Calcium carbonate and vitamin $\mathrm{D}$ were added to the patient's regimen to reduce his risk of osteoporosis. He was discharged on the seventh hospital day. Over 4 weeks, there was complete reversal of both cranial nerve palsies and also relief of the headaches associated with the arteritis and the cluster headaches. Prednisone therapy was tapered gradually over the subsequent 6 months and then changed to dexamethasone (1.5 mg per day) because of cushingoid symptoms and, despite the preventive measures taken, development of osteoporosis, with collapse of the 12 th thoracic vertebrae.

\section{Discussion}

The majority of patients who present with temporal arteritis complain of headache. The pain may be unilateral and often is localized to the affected scalp vessels. Pain in the jaw or temporal area during chewing is a pathognomonic symptom and is often the first evidence of the disorder. Characteristically described as a burning sensation, the pain also may be boring, sharp, or lancinating. Systemic symptoms, including weight loss, night sweats, and low-grade fever, may be present. Arthralgia and myalgia may occur in the patient who experiences temporal arteritis as a component of polymyalgia rheumatica. These patients may report the weak- ness as difficulty in rising out of a chair. Proximal joints and muscles are affected most commonly.

Other arteries besides those of the scalp may be involved in temporal arteritis. Because any medium to large artery may be involved in this disorder, a variety of presentations have been described. These include facial neuralgia, mental changes, ischemic cardiac disease, ear pain, vertigo, and deafness, as well as the more common involvement of the ophthalmic artery, which is responsible for loss of vision.

Laboratory studies commonly are used to help guide the diagnosis and treatment of temporal arteritis. The ESR, as determined by the Westergren method, is one of the most common parameters. A rate greater than $40 \mathrm{~mm} / \mathrm{h}$ is found in most patients, although the test may be normal in a significant percentage. ${ }^{2}$

Corticosteroids are the keystone of treatment in temporal arteritis. Prompt initiation of $60 \mathrm{mg}$ per day of prednisone, or the equivalent, will rapidly reverse the pain, fever, myalgia, and arthralgia. In about half of the cases, it also will prevent visual complications. Treatment should be initiated as soon as the disease is suspected clinically. The Westergren ESR should be determined prior to the first dose of corticosteroids. Biopsy of the involved superficial temporal artery should be performed when clinically indicated. In some cases, angiography of the extracranial vessels may help to guide the surgical procedure by identifying involved areas and, also, by avoiding "skip" lesions in the involved area, which can result in a negative biopsy in the patient with active disease.

In addition to the clinical picture, the ESR may prove useful on followup in guiding the reduction in prednisone dosage to maintenance levels of 10 to $20 \mathrm{mg}$ per day. Reduction of the corticosteroid dosage toward maintenance levels should be accomplished as soon as there is quiescence of the disease process. Prolonged use of corticosteroid agents is associated with weight gain, fluid retention, cataracts, diabetes mellitus, and osteoporosis.

In patients with proved temporal arteritis and a normal ESR, the sedimentation rate may become elevated later in treatment, in which case ESR levels may be used to monitor therapy. However, for many patients the clinical status is all that may be relied upon.

Even in classic cases of temporal arteritis, there is no consensus on the duration of corticosteroid therapy. Maintenance of a normal ESR and a clinically asymptomatic patient are minimum prerequisites to discontinuation of therapy. Even after the patient has no remaining symptoms and the ESR 
has returned to normal range, treatment with corticosteroids should be continued for at least 6 months. Based on the patient's response to treatment, therapy in some cases may need to be continued for up to 6 years. Despite prolonged therapy, the condition may become refractive to corticosteroid treatment and progress to the neurologic complications of temporal arteritis.

\section{Summary}

The neurologic complications of giant cell arteritis are typically irreversible. Prompt initiation of aggressive corticosteroid therapy may remove these deficits, although diagnostic studies such as the Westergren sedimentation rate provide a usual marker of the disease and its clinical progress in up to $30 \%$ of cases. Diagnostic evaluation provides the only key to initiation of corticosteroid and longterm management of the disease. $\overline{1 . \text { Horton BT, Magath TB, Brown GE: An undescribed form of arteritis }}$ of the temporal vessels. Mayo Clin Proc 1932;7:700-701.

2. Kansu T, Corbett JJ, Savino P, et al: Giant cell arteritis with normal sedimentation rate. Arch Neurol 1977;34:624-625.

3. Barricks ME, Traviesa DB, Glaser JS, et al: Ophthalmoplegia in cranial arteritis. Brain 1977;100:209-221.

4. Meadows SP: Temporal or giant cell arteritis in Smith, JL (ed): $O p h$ thalmic aspects in neuroophthalmology. St. Louis, CV Mosby, 1968, vol 4, pp 148-157.

5. Lipton RB, Solomon S, Wertenbaker C: Gradual loss and recovery of vision in temporal arteritis. Arch Intern Med 1985;145:2252-2253.

Dr. Freitag is associate director, Diamond Headache Clinic, Chicago, Ill, and visiting lecturer, Department of Family Medicine, Chicago College of Osteopathic Medicine, Chicago, Ill. Dr. Diamond is director, Diamond Headache Clinic, and adjunct professor of pharmacology, Chicago Medical School, North Chicago, Ill. Dr. Solomon is staff physician, Diamond Headache Clinic, and adjunct professor of pharmacology, Chicago Medical School.

Dr. Freitag, Diamond Headache Clinic, 5252 North Western Ave, Chicago, IL 60625. 